\title{
Perspectives for future neutrino oscillation experiments with accelerators: beams, detectors and physics
}

\author{
Pasquale Migliozzi $\dagger$ \\ † INFN, Sezione Napoli, Italy \\ E-mail: pasquale.migliozzi@cern.ch
}

\begin{abstract}
In recent years great progress toward the understanding of the mixing in the leptonic sector has been made. Nonetheless, this field of research is just at the beginning. Further advance by accelerator based neutrino oscillation experiments requires new beams and detectors to reach the wanted physics goals. In this paper we review the next possible steps that can be done for neutrino oscillation experiments with accelerators.
\end{abstract}

\section{A Brief Introduction to the Present Status of Neutrino Oscillation Searches}

The hypothesis of neutrino oscillations [1] is strongly supported by atmospheric [2, solar [3], accelerator [4] and reactor [5] neutrino data. If we do not consider the claimed evidence for oscillations by the LSND experiment [6], that must be confirmed or excluded by the ongoing MiniBooNE experiment [7], oscillations in the leptonic sector can be accommodated in the three family Pontecorvo-Maki-Nakagawa-Sakata (PMNS) mixing matrix $U_{P M N S}$

$$
U_{P M N S}=\left(\begin{array}{ccc}
c_{12} c_{13} & s_{12} c_{13} & s_{13} e^{-i \delta} \\
-s_{12} c_{23}-c_{12} s_{23} s_{13} e^{i \delta} & c_{12} c_{23}-s_{12} s_{23} s_{13} e^{i \delta} & s_{23} c_{13} \\
s_{12} s_{23}-c_{12} c_{23} s_{13} e^{i \delta} & -c_{12} s_{23}-s_{12} c_{23} s_{13} e^{i \delta} & c_{23} c_{13}
\end{array}\right)
$$

where the short-form notation $s_{i j} \equiv \sin \theta_{i j}, c_{i j} \equiv \cos \theta_{i j}$ is used. Further Majorana phases have not been introduced, since oscillation experiments are only sensitive to the two neutrino mass squared differences $\Delta m_{12}^{2}, \Delta m_{23}^{2}$ and to the four parameters in the mixing matrix of eq. (II): three angles and the Dirac CP-violating phase, $\delta$.

There are several global fits of all available data. As an example we report in Tab. [ the results taken from Ref. [8]. Notice that there are no direct measurements of the mixing angle $\theta_{13}$, but only the upper limit given by the CHOOZ reactor experiment 9 . The global analysis in Ref. [8] has given a bound on $\theta_{13}: \sin ^{2} \theta_{13} \leq 0.03$ at $90 \%$ C.L. 
Perspectives for future neutrino oscillation experiments with accelerators: beams, detectors and physics 2

\begin{tabular}{|c|c|c|c|c|}
\hline parameter & best fit & $2 \sigma$ & $3 \sigma$ & $5 \sigma$ \\
\hline$\Delta m_{21}^{2}\left[10^{-5} \mathrm{eV}^{2}\right]$ & 6.9 & $6.0-8.4$ & $5.4-9.5$ & $2.1-28$ \\
\hline$\Delta m_{31}^{2}\left[10^{-3} \mathrm{eV}^{2}\right]$ & 2.6 & $1.8-3.3$ & $1.4-3.7$ & $0.77-4.8$ \\
\hline $\sin ^{2} \theta_{12}$ & 0.30 & $0.25-0.36$ & $0.23-0.39$ & $0.17-0.48$ \\
\hline $\sin ^{2} \theta_{23}$ & 0.52 & $0.36-0.67$ & $0.31-0.72$ & $0.22-0.81$ \\
\hline
\end{tabular}

Table 1. Best-fit values, $2 \sigma, 3 \sigma$ and $5 \sigma$ intervals (1 d.o.f.) for the three-flavor neutrino oscillation parameters from global data including solar, atmospheric, reactor (KamLAND and CHOOZ) and accelerator (K2K) experiments.

The next steps on the way of a full understanding of neutrino oscillations by using neutrino beams produced at accelerators are

- confirm the source of atmospheric neutrino oscillations, i. e. observe the oscillation $\nu_{\mu} \rightarrow \nu_{\tau}$

- measure the remaining parameters of the PMNS mixing matrix: $\theta_{13}$ and $\delta$;

- measure the sign of $\Delta m_{23}^{2}$;

- perform precision measurements of the angles $\theta_{12}$ and $\theta_{23}$, and of $\Delta m_{12}^{2}$ and $\Delta m_{23}^{2}$.

It is worth noting that there are other searches (like $\beta$-decay and double- $\beta$ decay experiments, and space experiments studying anisotropies in cosmic background radiation) which provide very important information like the absolute value of the neutrino mass or whether the neutrino is a Dirac or a Majorana particle. For a comprehensive review of these experiments we refer to [10].

\section{Neutrino Beams}

Current neutrino oscillation experiments are based on beams where neutrinos come from the decay of mesons produced in the interaction of high energy protons impinging onto a target (typically Be or graphite). However, such conventional beams have some limitations (see Section 2.1) that could be overcome by using new beam-line concepts: $\beta$ beams (Section 2.2) or Neutrino Factories (Section 2.3). For a comprehensive discussion of future beams and their comparison we refer to [11] and references therein.

\subsection{Conventional Neutrino Beams}

One can identify the main components of a conventional neutrino beam line at a high energy accelerator as

- the target onto which protons are sent to produce pions and kaons;

- the focusing system which guides the mesons along the desired neutrino beam direction;

- the decay tunnel (usually evacuated) where mesons decay and produce neutrinos and muons. 
Perspectives for future neutrino oscillation experiments with accelerators: beams, detectors and physics3

From meson decay kinematics it follows that the neutrino energy is given by

$$
E_{\nu}=\frac{m_{\pi(K)}^{2}-m_{\mu}^{2}}{m_{\pi(K)}^{2}} \frac{E_{\pi(K)}}{\left(1+\gamma^{2} \theta^{2}\right)}
$$

where $\gamma$ is the Lorentz boost of the parent meson, $E_{\pi(K)}$ its energy and $\theta$ the angle of the neutrino with respect to the meson flight direction.

There are three types of conventional neutrino beams: the Wide Band Beams (WBB), the Narrow Band Beams (NBB) and the Off-Axis Beams (OAB). WBB are characterized by a wide energy spectrum (they could spread over a couple of order of magnitude) and correspondingly high neutrino flux. Given these features, WBB are the optimal solution to make discoveries. The drawback is that, if the signal comes from a small part of the energy spectrum, it could be overwhelmed by the background also induced by neutrinos outside the signal region. Conversely, NBB may produce almost monochromatic energy spectra. This can be obtained by selecting a small momentum bite of the parent $\pi$ and $K$. However, the neutrino yield is significantly reduced. This is an important drawback for oscillation searches.

A good compromise between the requirements of a high flux and a narrow energy spectrum is obtained by means of Off-Axis Beams [12. This technique involves designing a beam-line which can produce and focus a wide range of mesons in a given direction (as in the WBB case), but then putting the detectors at an angle with respect to that direction. Since the pion decay is a two-body decay, a given angle between the pion direction and the detector location corresponds to a given neutrino energy (almost) independently of the pion energy. Furthermore, the smaller fraction of high energy tails reduces the background from neutral-current $(\mathrm{NC})$ events, which can be mistaken for a $\nu_{e}$ charged-current (CC) interaction due to the early showering of gamma's from the $\pi^{0}$ decay.

It is worth noting that, independent of the adopted solution, there are common problems to all conventional neutrino beams

- the hadron yield in the proton-target interaction has large uncertainties due to lack of data and to theoretical difficulties in describing hadronic processes. This implies difficulties in predicting the neutrino flux and spectrum with good accuracy;

- in addition to the dominant flavor in the beam (typically $\nu_{\mu}$ ) there is a contamination (at the few percent level) from other flavors $\left(\bar{\nu}_{\mu}, \nu_{e}\right.$ and $\left.\bar{\nu}_{e}\right)$.

The knowledge of the beam spectrum and composition has a strong impact both on the precision measurements of the angle $\theta_{23}$, on the mass squared difference $\Delta m_{23}^{2}$ and on the sensitivity to the mixing angle $\theta_{13}$. For instance, from the CHOOZ limit on $\theta_{13}$ we know that the $\nu_{\mu} \rightarrow \nu_{e}$ appearance probability is smaller than $5 \%$, which is of the same order of magnitude of the beam contamination. Therefore, the observation of $\nu_{e}$ appearance and the related $\theta_{13}$ measurement are experimentally hard. The experimental problem related to the knowledge of the beam, namely the usage of a close detector, is addressed later in this Section. 

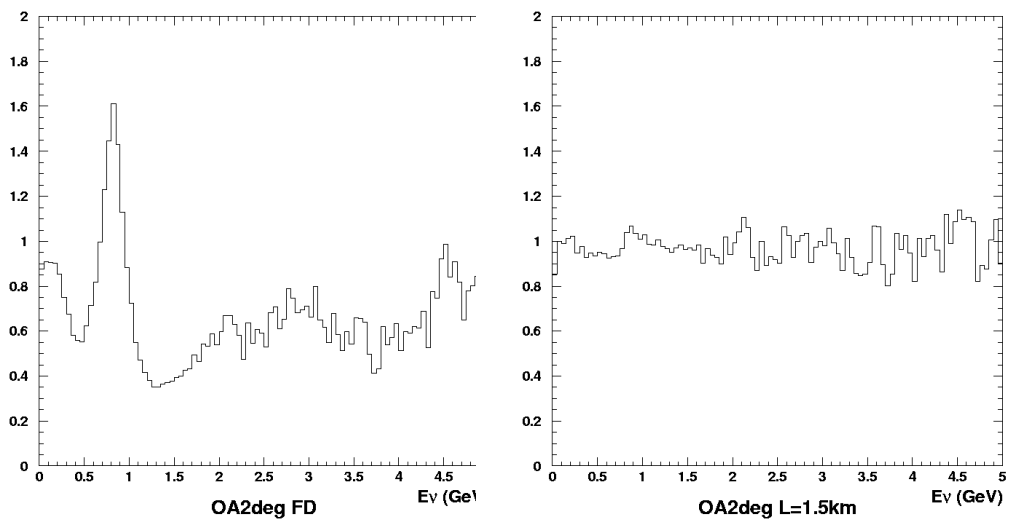

Figure 1. Far to close ratio as function of the close detector distance from the production target [13]: left panel $0.28 \mathrm{~km}$, right panel $1.5 \mathrm{~km}$.

In the last years a new concept of conventional beam (the so-called "Super-Beam") has been put forward in order to maximize the sensitivity to $\theta_{13}$. Super-Beams will provide a much higher neutrino flux, but at low energy (below $1 \mathrm{GeV}$ ). This will open the possibility to perform long-baseline experiments with high statistics and tuned at the oscillation maximum even at moderate distances between source and detector.

In order to improve the knowledge of the beam, conventional beam-lines are usually equipped with close detectors whose aim is to help in the prediction of the flux and spectrum of the neutrino beam in absence of oscillations (close to far extrapolation). In addition, they measure the intrinsic contamination (in particular the $\nu_{e}$ and $\bar{\nu}_{e}$ components), the background rejection capabilities of the detector and the neutrino cross-sections with high statistics.

Recently the J-PARC Collaboration pointed out, profiting of the experience gathered within the K2K Collaboration, that a single close detector is not sufficient to achieve systematic errors on the knowledge of the beam at the far detector better than $\sim 10 \%$. Therefore, they proposed to build two close detectors: one located at $250 \mathrm{~m}$ from the production target and a second at about $2 \mathrm{~km}$. Indeed, it turned out that at $2 \mathrm{~km}$ from the production target, the spectrum becomes almost identical to the one for Super-Kamiokande at $295 \mathrm{~km}$ distance. The far to close ratio as a function of the close detector distance is shown in Fig. 1. The ratio of the spectra at $295 \mathrm{~km}$ and $2 \mathrm{~km}$ deviate from unity only about 5\% [14. The small spectrum correction enables high precision prediction of far spectrum from the observation at the close detector. Consequently, it is possible to perform high precision measurements of the oscillation parameters.

\section{2. $\beta$-beams}

A $\beta$-beam [15] is made by accelerating radioactive ions with a short beta-decay lifetime, by storing them in a ring with straight sections and by letting them decay. The focusing 
of the beam is provided by the Lorentz boost. Having the possibility to accelerate either $\beta^{-}$(e.g. $\left.{ }^{6} \mathrm{He}\right)$ or $\beta^{+}$(e.g. ${ }^{18} \mathrm{Ne}$ ) ions, pure $\bar{\nu}_{e}$ or pure $\nu_{e}$ beams can be produced, respectively. In order to illustrate the value of the $\beta$-beam concept, we briefly discuss the production of an anti-neutrino beam. A good beta-emitter for anti-neutrino production is the ${ }^{6} \mathrm{He}^{++}$ion that decays into ${ }_{3}^{6} \mathrm{Li}^{++} e^{-} \bar{\nu}_{e}$ with a $\beta$-decay endpoint $\left(E_{0}\right)$ of about 3.5 MeV. The anti-neutrino spectrum is precisely known from laboratory measurements of the associated electron, since $E_{e}+E_{\nu} \approx E_{0}$. Since the ion is spin-less, decays at rest are isotropic. When ions are accelerated ( $\gamma$ values up to 150 are possible) the neutrino transverse momentum in the laboratory frame is identical to that observed in the rest frame, while the longitudinal momentum is multiplied by a factor $\gamma$. Therefore, neutrino beam divergence is of the order of $1 / \gamma$ (less than $10 \mathrm{mrad}$ for $\gamma=100$ ), and the average neutrino energy in the forward direction is $2 \gamma \mathrm{E}_{c m s} \sim 500 \mathrm{MeV}$.

The technical feasibility of accelerating ions, although at relatively low energies, has been already demonstrated in nuclear physics experiments such as at ISOLDE at CERN. Given the small neutrino energy, a potential drawback of this approach is the substantial background from atmospheric neutrinos. To overcome this problem, ion beams should be bunched. At present, this is a major technical issue.

Summarizing, the main features of a neutrino beam based on the $\beta$-beam concept are

- the neutrino beam energy is low and neutrinos are well focused (particularly important for long-baseline experiments);

- the beam energy depends on the $\gamma$ factor. The ion accelerator can be tuned to optimize the sensitivity of the experiment;

- the neutrino beam contains a single flavor with an energy spectrum and intensity known a priori. Therefore, unlike conventional neutrino beams, close detectors are not necessary;

- neutrino and anti-neutrino beams can be produced with a comparable flux.

\subsection{Neutrino Factory}

The first stage of a Neutrino Factory is similar to that of a Super-Beam. Namely, protons are sent onto a target producing pions and kaons that are collected by means of magnetic lenses. However, while in those beams hadrons are let decay launching neutrinos toward the detector site, in a Neutrino Factory daughter muons are collected and accelerated in a ring with long straight sections. Muon decays in each straight section generate highly collimated neutrino beams. If $\mu^{+}$are stored, $\mu^{+} \rightarrow e^{+} \nu_{e} \bar{\nu}_{\mu}$ decays generate a beam consisting of $50 \% \nu_{e}$ and $50 \% \bar{\nu}_{\mu}$. Similarly, if $\mu^{-}$are stored the beam consists of $50 \% \nu_{\mu}$ and $50 \% \bar{\nu}_{e}$. Since the kinematics of muon decay is well known, we expect minimal systematic uncertainties on the neutrino flux and spectrum. Hence, compared to conventional neutrino beams, Neutrino Factories provide $\nu_{e}$ and $\bar{\nu}_{\mu}$ beams or $\nu_{\mu}$ and $\bar{\nu}_{e}$ beams, with small systematic uncertainties on the flux and spectrum. Radiative effects 
Perspectives for future neutrino oscillation experiments with accelerators: beams, detectors and physics6

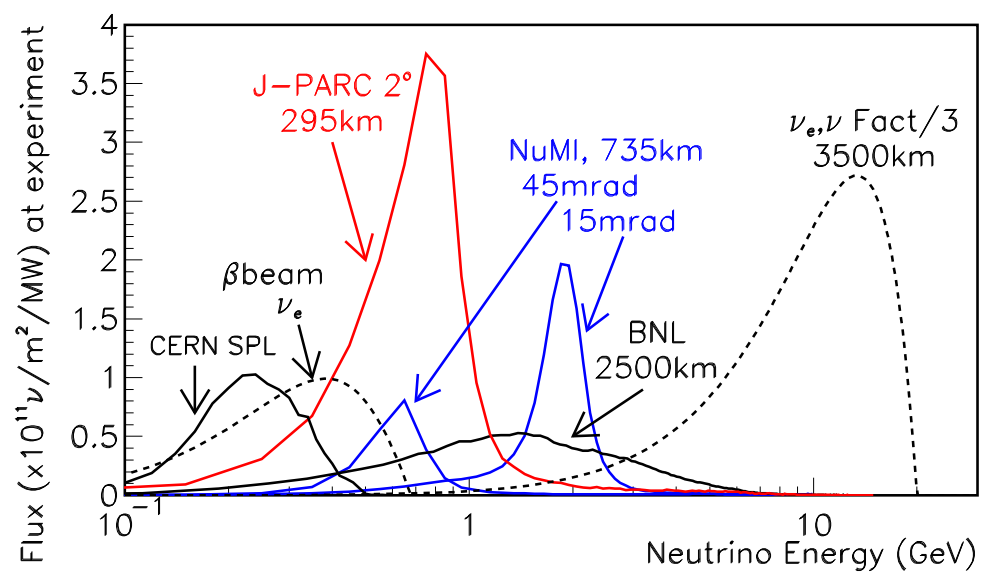

Figure 2. Neutrino fluxes for some of the beam-lines discussed in the previous Sections (from Ref. [17])

on the muon decay have been calculated and amount to about $4 \times 10^{-3}$ with a much smaller error. Overall, the flux is expected to be known with a precision of the order of $10^{-3}$. Another important feature of a Neutrino Factory beam is its sharp cut-off at the energy of the stored muons. In a conventional neutrino beam there is a high-energy tail which, as already mentioned, gives rise to background from $\mathrm{NC}$ events in which a leading $\pi^{0}$ is misinterpreted as an electron, faking $\nu_{\mu} \rightarrow \nu_{e}$ signal. Furthermore, the possibility to store high-energy muons that in turn produce high-energy neutrinos opens the study of oscillation channels like $\nu_{\mu} \rightarrow \nu_{\tau}$ and $\nu_{e} \rightarrow \nu_{\tau}$, whose combined physics potential has been discussed in [16].

\subsection{Beam-line Summary}

The expected fluxes for some of the future proposed beam-lines are shown in Fig. 20 [17]. The fluxes are normalized to $1 \mathrm{MW}$ proton power, except for the $\beta$-beam, which uses considerably less proton power. Notice that the fluxes are given at the distance where the detector is expected to be located.

\section{Detector Technologies}

\subsection{Water Cerenkov Detector}

After the observations of solar and supernovae neutrinos, the discovery of neutrino oscillations in the atmospheric sector represents another great success of the Cerenkov technique. The main advantages of water Cerenkov detectors are both economical and scientific. The target material and the possibility to instrument only the surface of the target make these detectors relatively cheap. The charged leptons are identified through the detection of Cerenkov light. The features of the ring are used for particle identification. A muon crossing the detector scatters very little. Therefore, the 
associated ring has very sharp edges. Conversely, an electron scatters (showers) much more, producing rings with "fuzzy" edges. The efficiency to identify an electron or a muon is larger than $99 \%$ for both leptons. The total measured light gives an estimate of the lepton energy, while the time measurement provided by each photomultiplier determines the outgoing lepton direction. By combining all this information it is possible to fully reconstruct the energy, the direction and the flavor of the incoming neutrino. It is worth noting that the procedure discussed above is suitable only for quasi-elastic events $\left(\nu_{l} n \rightarrow l^{-} p\right)$. Indeed, for non quasi-elastic events there are other particles in the final state, carrying a large energy fraction, that either are below the Cerenkov threshold or are neutrals. Therefore, the total neutrino energy is not very well measured. Furthermore, the presence of more than one particle above threshold produces more than one ring, spoiling the particle identification capability of the detector.

Recently, a Cerenkov detector using heavy water as target became operational (SNO). Besides the features discussed above, the SNO detector is also able to identify NC neutrino interactions (see Ref 18 for details) through the detection of the neutron produced in the reaction $\nu_{l} d \rightarrow \nu_{l} p n$. Thanks to the SNO results on the measurement of NC interactions of neutrinos coming from the Sun, it has been possible to clarify the longstanding solar neutrino problem. Namely, it has been proved that electron neutrinos produced in the Sun oscillate into active neutrinos (muon or tau).

Given its cost effectiveness and its excellent performance at low neutrino energies, the Cerenkov technique is often considered to operate at the future neutrino beam-lines providing low energy beams.

\subsection{Magnetized Iron Calorimeter}

Magnetized iron calorimeters are used since the eighties. The MINOS Collaboration has built a magnetized iron calorimeter to study neutrino oscillations at the atmospheric scale by using the NuMI long-baseline beam [19]. We use the MINOS performance as a reference for this kind of technology. The detector is composed of $2.54 \mathrm{~cm}$-thick steel planes interspersed with planes of $1 \mathrm{~cm}$-thick and $4.1 \mathrm{~cm}$-wide scintillator strips. The iron is magnetized to an average field of about 1.5 T. Simulations, as well as test beam results, show that the energy resolution of this tracking calorimeter is $55 \% / \sqrt{E(\mathrm{GeV})}$ and $23 \% / \sqrt{E(\mathrm{GeV})}$ for hadronic and electromagnetic showers, respectively. This technology is particularly suited for the reconstruction $\nu_{\mu} \mathrm{CC}$ events, while the electron identification is rather poor. Therefore, magnetized iron calorimeters are planned to be used to study either $\nu_{\mu}$ appearance in a pure $\nu_{e}$ beam or $\nu_{\mu}$ disappearance in a well known $\nu_{\mu}$ beam. It is worth noting that the presence of the magnetic field is essential in order to tag the (anti-)neutrino in the final state. This technology has been proposed to study the so-called "golden channels" $\nu_{e} \rightarrow \nu_{\mu}$ and $\bar{\nu}_{e} \rightarrow \bar{\nu}_{\mu}$ [20] at a Neutrino Factory. 
Perspectives for future neutrino oscillation experiments with accelerators: beams, detectors and physics 8

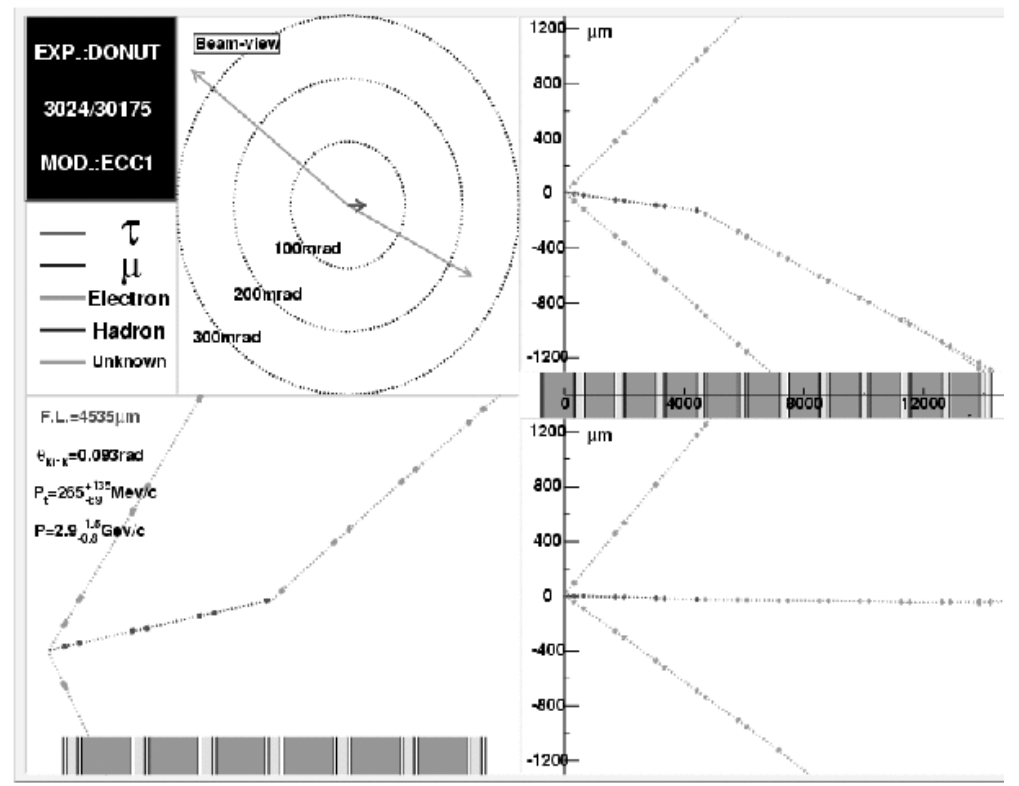

Figure 3. Schematic view of a $\tau$ decay candidate observed in the ECC of the DONUT experiment.

\subsection{Hybrid Emulsion Detector}

The Emulsion Cloud Chamber (ECC) concept (see references quoted in 21]) combines the high-precision tracking capabilities of nuclear emulsions and the large mass achievable by employing metal plates as a target. It has been adopted by the OPERA Collaboration 21] for a long-baseline search for $\nu_{\mu} \rightarrow \nu_{\tau}$ oscillations in the CNGS beam through the direct detection of the decay of the $\tau$ produced in $\nu_{\tau} \mathrm{CC}$ interactions. As an example of $\tau$ detection, we show in Fig. [3 one of the $\nu_{\tau}$ events observed in the DONUT experiment [22].

The basic element of the OPERA ECC is a "cell" made of a $1 \mathrm{~mm}$ thick lead plate followed by a thin emulsion film. The film consists of $44 \mu \mathrm{m}$-thick emulsion layers on either side of a $200 \mu \mathrm{m}$ plastic base. The number of grains hits in each emulsion layer (15-20) ensures redundancy in the measurement of particle trajectories and allows the measurement of their energy loss that, in the non-relativistic regime, can help to distinguish between different mass hypotheses.

Thanks to the dense ECC structure and to the high granularity provided by the nuclear emulsions, the OPERA detector is also suited for electron and $\gamma$ detection. The resolution in measuring the energy of an electromagnetic shower is about 20\%. Nuclear emulsions are able to measure the number of grains associated to each track. This allows an excellent two-track separation: $\mathcal{O}(1 \mu \mathrm{m})$ or even better. Therefore, it is possible to disentangle single-electron tracks from electron pairs coming from $\gamma$ conversion in the lead.

The outstanding position resolution of nuclear emulsions can also be used to measure the angle of each track segment with an accuracy of about 1 mrad. This allows 
Perspectives for future neutrino oscillation experiments with accelerators: beams, detectors and physics9

a momentum measurement by using multiple Coulomb scattering, with a resolution of about $20 \%$, and the reconstruction of kinematical variables characterizing the event.

A lead-emulsion detector has been also proposed to operate at a Neutrino Factory to study the so-called silver channel $\nu_{e} \rightarrow \nu_{\tau}[16]$.

\subsection{Liquid Argon Time Projection Chamber}

The ICARUS Collaboration [23] has developed a liquid argon time projection chamber (TPC). It is continuously sensitive and self-triggering, with the ability to provide threedimensional imaging of ionizing tracks.

The operating principle is rather simple: any ionizing event (from a particle decay or interaction) taking place in the active liquid argon volume, which is maintained at a temperature $T \sim 89 \mathrm{~K}$, produces ion-electron pairs. In the presence of a strong electric field $(\sim 0.5 \mathrm{KeV} / \mathrm{cm})$, the ions and electrons drift. The motion of the faster electrons induces a current on a wire plane located near the end of the sensitive volume. The electrons are collected by a wire plane with a different orientation. The knowledge of the wire positions and the drift time provides the three-dimensional image of the track, while the charge collected on the wires provides information on the deposited energy.

The detector consists of a large vessel of liquid argon filled with planes of wires strung on the different orientations. This device allows tracking, $d E / d x$ measurements and a full-sampling electromagnetic and hadronic calorimetry. Furthermore, the imaging provides excellent electron and photon identification and electron/hadron separation. The energy resolution is excellent for electromagnetic showers $(\sim 13 \% / \sqrt{E(\mathrm{MeV})})$ and also very good for contained hadronic showers $(30 \% / \sqrt{E(\mathrm{GeV})})$. Furthermore, it is possible to measure the momentum of stopping muons with a resolution better than $3 \%$, by using the Multiple Coulomb Scattering.

The major milestone of this technique has been the successful operation of the ICARUS 600 tons prototype which has operated during the summer of 2001. An event recorded with the 600 tons detector is shown in Fig. 4. Of course neutrino oscillation studies require an increase of the total liquid argon mass. A 2.5 kton detector is foreseen to operate with the CNGS neutrino beam to search for $\nu_{\mu} \rightarrow \nu_{\tau}$ oscillations at the atmospheric scale. Given its excellent electron identification capabilities, it has been also proposed to operate on other beams to search for $\nu_{\mu} \rightarrow \nu_{e}$ appearance.

\subsection{Low Z Calorimeter}

Unlike the iron calorimeters discussed in Section 3.2. low Z calorimeters allow a good identification and energy measurement of electrons produced in $\nu_{e} \mathrm{CC}$ interactions. In fact, for this purpose one must sample showers more frequently than about $1.4 \mathrm{X}_{0}$ and a magnetic field is not necessary. Another advantage of a low $\mathrm{Z}$ calorimeter is that, for a given sampling in units of radiation length, one can have up to a factor 3 more mass per readout plane with respect to iron calorimeters. This detector can discriminate between $\mathrm{NC}$ and $\nu_{e}$ induced $\mathrm{CC}$ events by looking at the longitudinal profile of the 


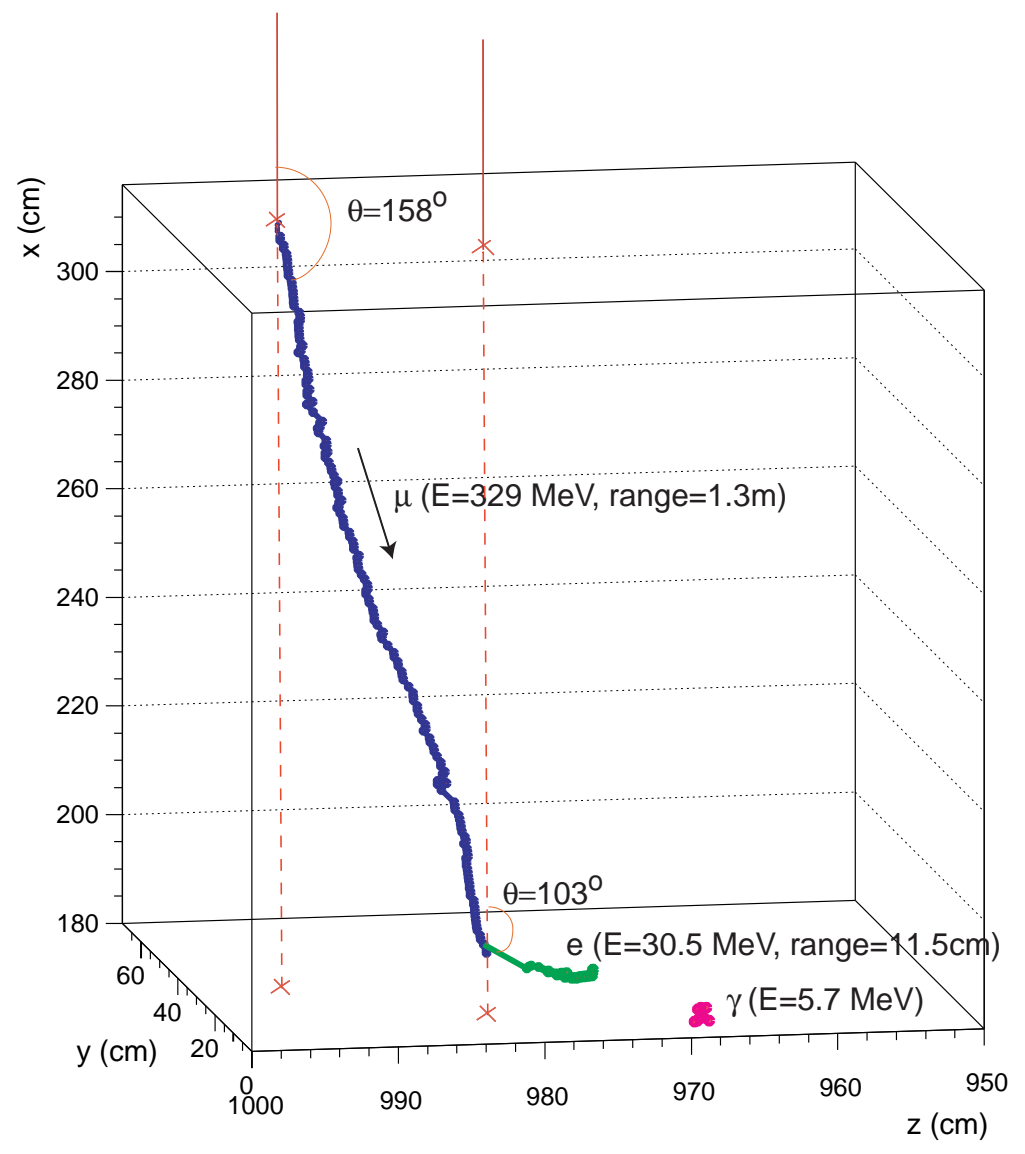

Figure 4. Stopping muon in the ICARUS 600 tons detector and decaying into an electron.

neutrino interaction, as $\mathrm{NC}$ events are likely to be much more spread out in the detector than $\nu_{e}$ CC. Several active detectors (Resistive Plate Chambers, streamer tubes, plastic scintillators) have been considered and are currently under investigation. This technique has been proposed to search for $\nu_{\mu} \rightarrow \nu_{e}$ oscillations in the NUMI Off-Axis beam-line [27.

\section{Physics Reach of Future Accelerator-Based Oscillation Experiments}

\subsection{Near Term Programs}

The NuMI (expected to start beginning 2005) and the CNGS (expected to start mid 2006) programs were approved with the aim to search for neutrino oscillation in the $\Delta m^{2}$ region indicated by the atmospheric neutrino results. By looking at the $\nu_{\mu}$ disappearance in an almost pure $\nu_{\mu}$ beam, the MINOS experiment 24] at NuMI aims at the measurement of the oscillation parameters with a precision of about $10 \%$. A statistical evidence of $\nu_{\mu} \rightarrow \nu_{\tau}$ oscillations is achievable by looking at the $\mathrm{NC} / \mathrm{CC}$ ratio. On the other hand, the CNGS program aims at the direct evidence of $\nu_{\tau}$ appearance in a pure $\nu_{\mu}$ beam by searching for the decays of $\tau$ produced in $\nu_{\tau} \mathrm{CC}$ interactions. 
After five years of data taking and with $\Delta m_{23}^{2}=2 \times 10^{-3} \mathrm{eV}^{2}$, about 10 events, with a background smaller than 1 event, are expected both in ICARUS and OPERA [25]. A $50 \%$ increase of the proton intensity [28], with a corresponding increase of the event rate, is envisaged. These experiments are also able to search for $\nu_{\mu} \rightarrow \nu_{e}$ oscillations, i.e. a non vanishing $\theta_{13}$. It has been shown in Ref. [29, 30] that in case of negative result, the CNGS program (ICARUS and OPERA together) will be able to exclude values of $\theta_{13}$ down to $5^{\circ}$ at $90 \%$ C.L., to be compared with the $\theta_{13}<10^{\circ}$ limit given by CHOOZ. On the other hand, if $\theta_{13}$ is larger than $7^{\circ}$ it will be possible to provide a first measurement of $\theta_{13}$.

\subsection{Medium Term Programs}

The main goals of the J-PARC [26] (expected to start in 2008-09) and NuMI-OA [27] (start of data taking after 2010) programs are to provide precision measurements of the PMNS matrix elements relevant in the atmospheric sector with a few percent accuracy and to push the sensitivity to $\theta_{13}$ down to a few degrees, i.e. improve the CHOOZ limit by one order of magnitude. As far as the detector is concerned, the J-PARC project will exploit the Super-Kamiokande detector located about $300 \mathrm{~km}$ far from the neutrino source under construction at the Tokai site in Japan. The NuMI-OA project will exploit the NuMI beam with a low $\mathrm{Z}$ calorimeter located at a distance not yet defined, but in the 700 - $900 \mathrm{~km}$ range.

It is worth to stress that the information gathered from the combination of results from different experiments is actually better than if one had simply run a single experiment longer [31]. The reason for this is that NuMI-OA experiment can be sensitive to matter effects, while J-PARC experiment is not.

\subsection{Long Term Programs}

The main goal of future projects like Super-Beams (i.e. J-PARC-II [32], Super AGS [33, SPL [34, 11]), $\beta$-beams or Neutrino Factories is the discovery and the measurement of the $\mathrm{CP}$ violating phase $\delta$ in the leptonic sector. This search is very challenging and intrinsically complex. As an example, we report about the search for $\delta$ at a Neutrino Factory.

The transition probabilities $\nu_{e} \rightarrow \nu_{\mu}$ and $\bar{\nu}_{e} \rightarrow \bar{\nu}_{\mu}$ are extremely sensitive to $\theta_{13}$ and $\delta$ : these are the so-called "golden measurements" [20] and can be studied by searching for wrong-sign muons, provided the considered detector has a good muon charge identification capability. The determination of $\left(\theta_{13}, \delta\right)$ from this channel is not free of ambiguities: it was shown in 35] that, for a given physical input parameter pair $\left(\bar{\theta}_{13}, \bar{\delta}\right)$, measurements of the oscillation probability for $\nu_{e} \rightarrow \nu_{\mu}$ and $\bar{\nu}_{e} \rightarrow \bar{\nu}_{\mu}$ generally result in two allowed regions of the parameter space. Worse than that, further degeneracies results from our ignorance of the sign of $\Delta m_{23}^{2}$ and from the approximate $\left[\theta_{23}, \pi / 2-\theta_{23}\right]$ symmetry for the atmospheric angle [36, 37]. In general, the measurement 

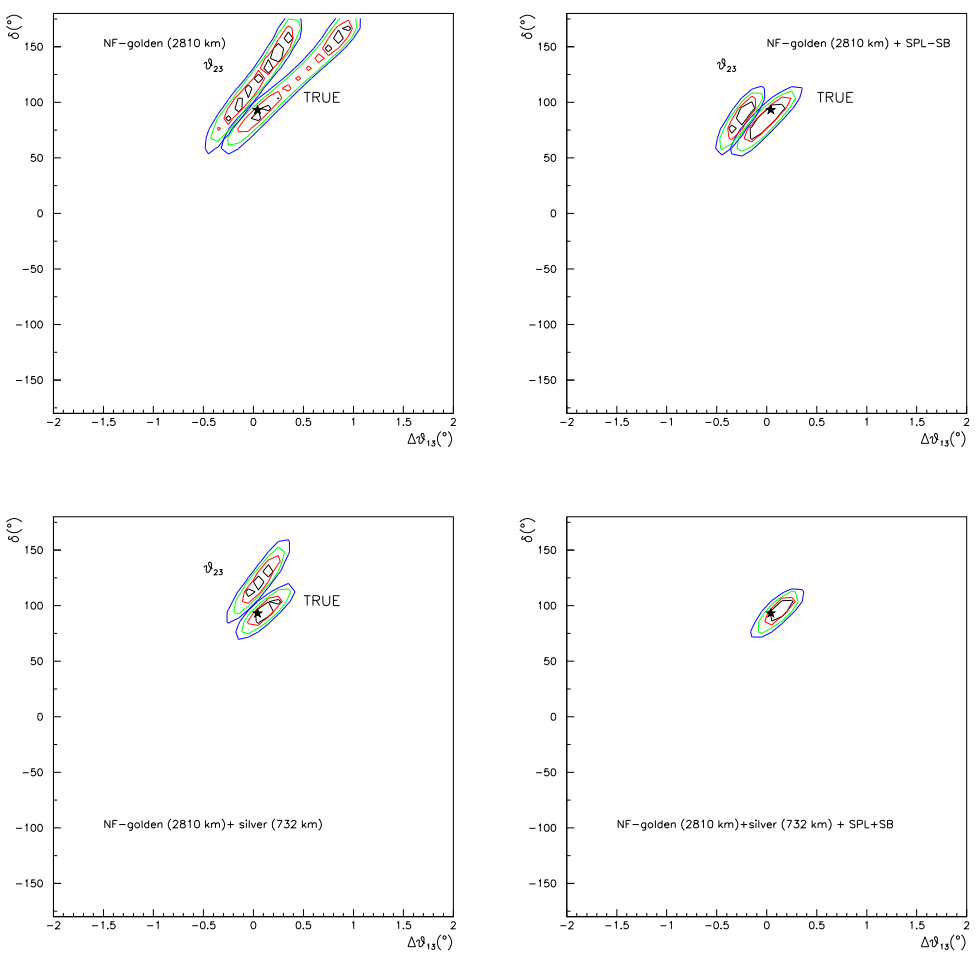

Figure 5. The results of a $\chi^{2}$ fit for $\bar{\theta}_{13}=2^{\circ} ; \bar{\delta}=90^{\circ}$. Four different combinations of experimental data are presented: a) magnetized iron detector (MID) at a Neutrino Factory (NF); b) MID at NF plus water Cerenkov (WC) at a SPL; c) MID plus hybrid emulsion (HE) at NF; d) the three detectors together. Notice how in case (d) the eightfold-degeneracy is solved and a good reconstruction of the physical $\theta_{13}, \delta$ values is achieved.

of $P\left(\nu_{e} \rightarrow \nu_{\mu}\right)$ and $P\left(\bar{\nu}_{e} \rightarrow \bar{\nu}_{\mu}\right)$ will result in eight allowed regions of the parameter space, the so-called eightfold-degeneracy [37].

In order to solve these ambiguities, a single experiment on a single neutrino beam is not enough. An optimal combination of $\beta$-beams, Super-Beams and Neutrino Factories has to be considered to deal with the eightfold degeneracy. Several investigations on how to solve this problem have been carried out, as reported in 38] and references therein. As an example we report in Fig. 5 (taken from [38) the result of an analysis which combines a magnetized iron detector and a hybrid emulsion detector studying at a Neutrino Factory the golden and the silver channels, respectively, and a water Cerenkov detector exposed to a Super-Beam. For comparison, results achievable by using a combination of only two detectors or by the magnetized iron detector alone are also given.

\subsection{The Implications of Present and Medium Term on the Long Term Programs}

Having illustrated the experimental programs toward a precision measurement of the PMNS matrix, some comments are in order (for more details we refer to 30]). One 

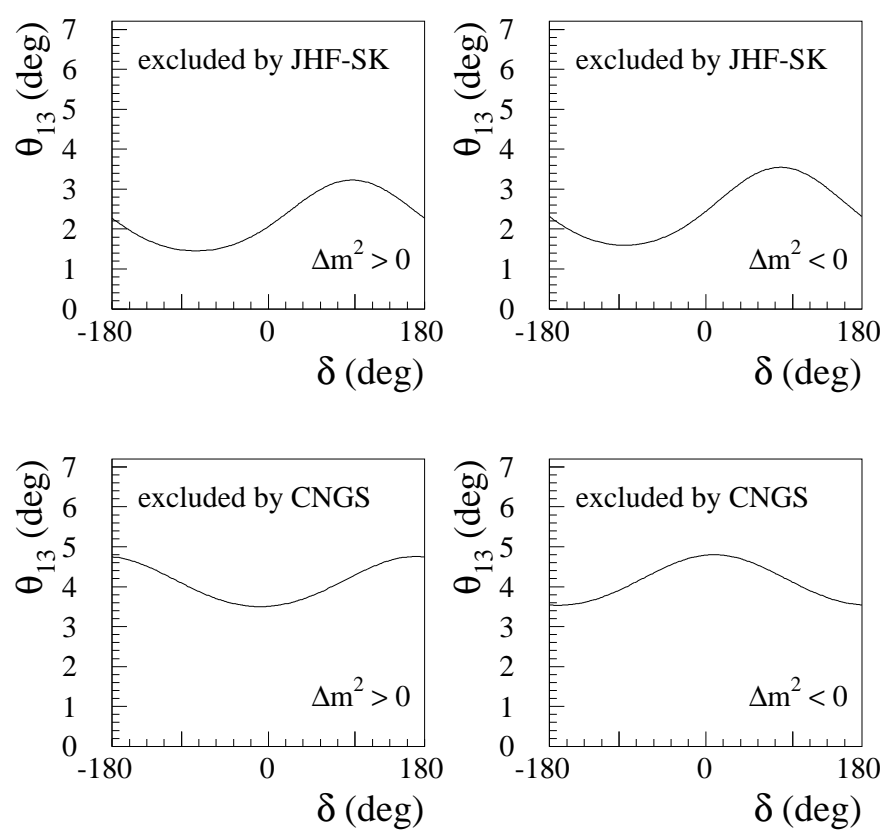

Figure 6. $\sin ^{2} 2 \theta_{13}$ sensitivity at $90 \%$ C.L. versus $\delta$ and for both positive and negative $\Delta m_{23}^{2}$. JHK-SK stands for J-PARC.

would like to approve and fund on firm basis expensive long term programs. The recent KAMLAND result strongly supports a high value of $\Delta m_{12}^{2}$ and a large, although not maximal, mixing angle. Therefore, this result places the long term program on a firmer ground since it guarantees that sub-dominant effects will not be suppressed to an unobservable level $\left(\alpha \equiv \Delta m_{12}^{2} / \Delta m_{23}^{2} \ll 10^{-2}\right)$. However, there is a second condition, which at present remains poorly constrained, to ensure that $\delta$ is observable: $\theta_{13}$ should not be vanishingly small.

We then expect present and medium term programs to measure $\theta_{13}$ in order to assess the possibility of exploring CP violation through Super-Beams (i.e. J-PARC-II, Super AGS, SPL), $\beta$-beams or Neutrino Factories. However, effects driven by $\theta_{13}$ are correlated with the ones driven by $\delta$. As an example we show in Fig. [6 the $\theta_{13}$ sensitivity at $90 \%$ C.L. as a function of $\delta$ for J-PARC and for CNGS [30. For positive values of $\delta$, the $\mathrm{CP}$ phase dependence of J-PARC has the worst possible behavior for the medium term program, since the minimum sensitivity to $\sin ^{2} 2 \theta_{13}$ is achieved at maximum $\left(90^{\circ}\right)$ $\mathrm{CP}$ violation phase (maximum discovery potential for long term programs).

The evaluation of the physics reach of the long term experiments will be particularly difficult if none of the medium term experiments finds a conclusive $\theta_{13}$ signal. It will be impossible to lift the $\theta_{13}-\delta$ correlation without additional external information; i.e. it is impossible to decide whether the lack of events is due to the smallness of $\theta_{13}$ 


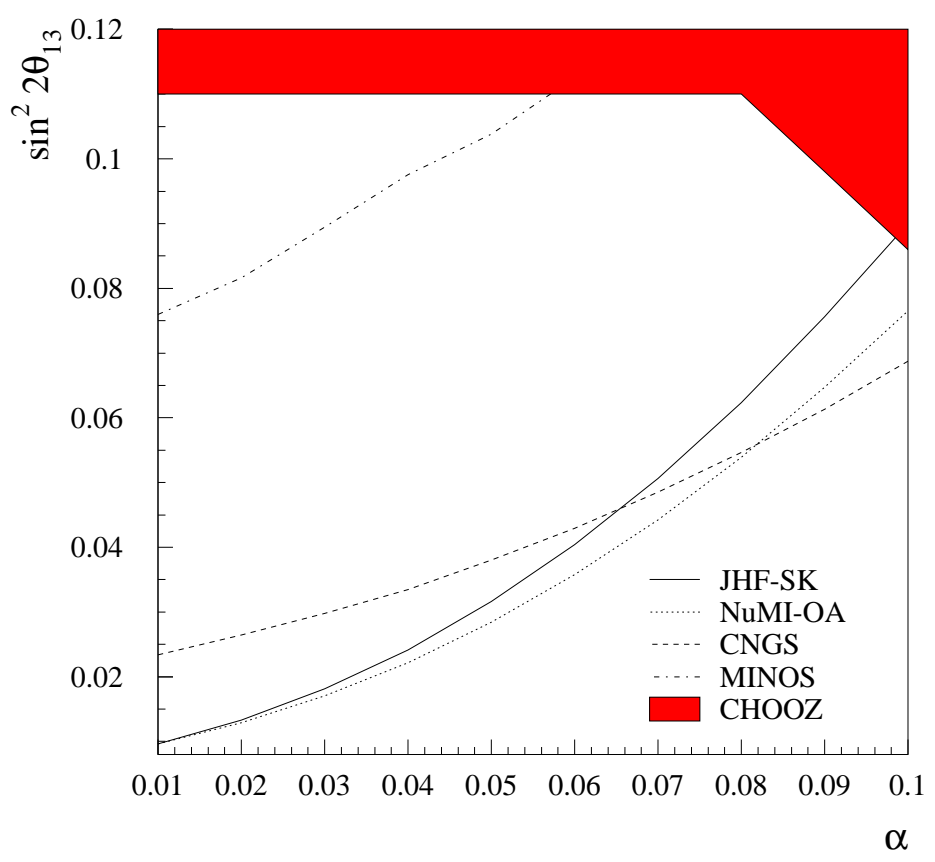

Figure 7. $\sin ^{2} 2 \theta_{13}$ sensitivity at $90 \%$ C.L. versus $\alpha \equiv \Delta m_{21}^{2} /\left|\Delta m_{31}^{2}\right|$. JHK-SK stands for J-PARC.

or it is the outcome of a cancellation effect between a large value of $\theta_{13}$ and a large value of $\delta$. Additional ambiguities are present when matter effects are appreciable. A careful optimization of the whole medium term program can considerably improve the sensitivity to $\theta_{13}$. Therefore, one should optimize the data taking in order to decouple the $\theta_{13}-\delta$ correlation. In particular, one could decide to run one of the neutrino beam-lines with anti-neutrinos.

The $\theta_{13}$ sensitivity achievable by each medium term experiement (and incorporating all the effect of degeneracies) is shown in Fig. 7 [30].

We can conclude that without an optimal running of all available beam-lines it will be impossible for present and medium term programs to ground (or discourage in a definitive manner) the building of long term facilities.

\section{Conclusion}

So far, neutrinos are the only source of physics beyond the Standard Model of particle physics: the neutrino oscillations discovery has unambiguously given evidence for a nonzero neutrino mass and shown the non-conservation of the leptonic number. However, there is a still long way to go before the mixing matrix in the leptonic sector and the masses are precisely determined. There are ongoing efforts to provide new neutrino sources (neutrino beams) and new detector technologies or upgrades of the existing 
Perspectives for future neutrino oscillation experiments with accelerators: beams, detectors and physics 15

\begin{tabular}{|c|c|c|c|c|}
\hline Program & Detector & $\begin{array}{c}<E> \\
(\mathrm{GeV})\end{array}$ & $\begin{array}{c}\mathrm{L} \\
(\mathrm{km})\end{array}$ & evts/kton/yr \\
\hline NuMI & MI & 3.5 & 730 & 469 \\
\hline CNGS & HE LAr & 18 & 732 & 2500 \\
\hline J-PARC & WC & 0.7 & 295 & 133 \\
\hline NuMI-OA & LZ & 2 & $700-900$ & 80 \\
\hline J-PARC-II & WC & 0.7 & 295 & 691 \\
\hline Super AGS & WC, LAr, LZ & 1.5 & 2540 & 11 \\
\hline SPL & WC, LAr & 0.26 & 130 & 16 \\
\hline$\beta$-beam & WC & 0.58 & 130 & 84 \\
\hline$\nu$-Factory & MI, HE, LAr & $10-30$ & $700-3000$ & $10^{5}-10^{3}$ \\
\hline
\end{tabular}

Table 2. Main features of future long base-line neutrino oscillation experiments based on accelerators and the proposed detector techniques. In the Table we used

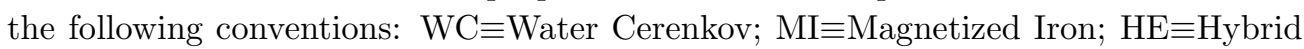
Emulsion; LAr $\equiv$ Liquid Argon; LZ $\equiv$ Low Z .

ones. An overview of all existing, under construction and proposed beam-lines and detectors is given in Table 2 .

Given these premises, one can think that in the future the year 1998 will remembered not only as the year of the neutrino oscillations discovery, but as the starting date of a new "mirabilis era" for neutrino physics.

[1] B. Pontecorvo, Sov. Phys. JETP 6 (1957) 429 [Zh. Eksp. Teor. Fiz. 33 (1957) 549];

Z. Maki, M. Nakagawa and S. Sakata, Prog. Theor. Phys. 28 (1962) 870;

B. Pontecorvo, Sov. Phys. JETP 26 (1968) 984;

V. N. Gribov and B. Pontecorvo, Phys. Lett. B 28 (1969) 493.

[2] Y. Fukuda et al. [Super-Kamiokande Coll.], Phys. Rev. Lett. 81, 1562 (1998);

M. Ambrosio et al. [MACRO Coll.], Phys. Lett. B517 (2001) 59.

[3] B.T. Cleveland et al., Astrophys. J. 496, 505 (1998);

J.N. Abdurashitov et al. [SAGE Coll.], Phys. Rev. C60, 055801 (1999);

W. Hampel et al. [GALLEX Coll.], Phys. Lett. B447, 127 (1999);

S. Fukuda et al. [Super-Kamiokande Coll.], Phys. Rev. Lett. 86, 5651 (2001);

Q.R. Ahmad et al. [SNO Coll.], Phys. Rev. Lett. 87, 071301 (2001).

[4] M.H. Ahn et al. [K2K Coll.], Phys. Rev. Lett. 90 (2003) 041801.

[5] K. Eguchi et al. [KamLAND Coll.], Phys. Rev. Lett. 90 (2003) 021802.

[6] C. Athanassopoulos et al. [LSND Collaboration], Phys. Rev. Lett. 81 (1998) 1774 [arXiv nucl-ex/9709006; A. Aguilar et al. [LSND Collaboration], Phys. Rev. D 64 (2001) 112007 [arXiv hep-ex/0104049.

[7] Details on the MiniBooNE detector are available at http://www-boone.fnal.gov/

[8] M. Maltoni, T. Schwetz, M. A. Tortola and J. W. Valle, arXiv hep-ph/0309130

[9] M. Apollonio et al. [CHOOZ Collaboration], Phys. Lett. B 466 (1999) 415 [arXiv hep-ex/9907037;

M. Apollonio et al., Eur. Phys. J. C 27 (2003) 331 [arXiv hep-ex/0301017.

[10] W. M. Alberico and S. M. Bilenky, arXiv hep-ph/0306239.

S. M. Bilenky, C. Giunti, J. A. Grifols and E. Masso, Phys. Rept. 379 (2003) 69 [arXiv hep-ph/0211462.

[11] M. Apollonio et al., arXiv hep-ph/0210192 
Perspectives for future neutrino oscillation experiments with accelerators: beams, detectors and physics16

[12] D. Beavis et al., BNL No. 52459, April 1995.

[13] Courtesy of T. Kajita.

[14] T. Kobayashi, Nucl. Phys. Proc. Suppl. 111 (2002) 163.

[15] P. Zucchelli, Phys. Lett. B 532 (2002) 166.

[16] A. Donini, D. Meloni and P. Migliozzi, Nucl. Phys. B 646 (2002) 321 [arXiv hep-ph/0206034.

D. Autiero et al., arXiv hep-ph/0305185

[17] D. A. Harris, FERMILAB-CONF-03-328-E

[18] J. Boger et al. [SNO Collaboration], Nucl. Instrum. Meth. A 449 (2000) 172 [arXiv nucl-ex/9910016.

[19] M. V. Diwan, "Invited talk at the Seventh International Workshop on Tau Lepton Physics (TAU02), Santa Cruz, Ca, USA, Sept 2002", arXiv hep-ex/0211026.

[20] A. Cervera, A. Donini, M. B. Gavela, J. J. Gomez Cadenas, P. Hernandez, O. Mena and S. Rigolin, Nucl. Phys. B 579 (2000) 17 [Erratum-ibid. B 593 (2001) 731] [arXiv hep-ph/0002108.

[21] M. Guler et al. [OPERA Collaboration], CERN-SPSC-2000-028;

K. Kodama et al. [OPERA Collaboration], CERN-SPSC-99-20.

[22] K. Kodama et al. [DONUT Collaboration], Phys. Lett. B 504 (2001) 218 [arXiv hep-ex/0012035.

K. Kodama et al., Nucl. Instrum. Meth. A 493 (2002) 45.

[23] P. Aprili et al. [ICARUS Collaboration], modules to reach the design sensitive mass. (Addendum)," CERN-SPSC-2002-027

[24] MINOS Technical Design Report NuMI-L-337 TDR, http://www-numi.fnal.gov:8875/minwork/info/tdr/, http://www-numi.fnal.gov:8875/forscientists.html

V. Paolone, Nucl. Phys. Proc. Suppl. 100 (2001) 197; S. G. Wojcicki, Nucl. Phys. Proc. Suppl. 91 (2001) 216.

[25] D. Duchesneau [OPERA Collaboration], eConf C0209101 (2002) TH09 [arXiv hep-ex/0209082.

[26] Y. Itow et al., arXiv hep-ex/0106019

[27] D. Ayres et al., arXiv hep-ex/0210005

[28] R. Cappi et al., CERN-PS-2001-041-AE.

[29] M. Komatsu, P. Migliozzi and F. Terranova, J. Phys. G 29 (2003) 443 [arXiv;hep-ph/0210043;

[30] P. Migliozzi and F. Terranova, Phys. Lett. B 563 (2003) 73 [arXiv hep-ph/0302274.

[31] P. Huber, M. Lindner and W. Winter, Nucl. Phys. B 654 (2003) 3 [arXiv hep-ph/0211300.

[32] M. Furusaka et al., JAERI/KEK Joint Project Proposal KEK-REPORT-99-4, JAERI-TECH-9901.

[33] M. V. Diwan et al., Phys. Rev. D 68 (2003) 012002 [arXiv/hep-ph/0303081.

[34] B. Autin et al., CERN report CERN-2000-012 (2000).

[35] J. Burguet-Castell, M. B. Gavela, J. J. Gomez-Cadenas, P. Hernandez and O. Mena, Nucl. Phys. B 608 (2001) 301 [arXiv hep-ph/0103258.

[36] H. Minakata and H. Nunokawa, JHEP 0110 (2001) 001 [arXiv hep-ph/0108085.

[37] V. Barger, D. Marfatia and K. Whisnant, Phys. Rev. D 65 (2002) 073023 [arXiv hep-ph/0112119.

[38] A. Donini, "NUFACT'03: The fate of the clones," arXiv/hep-ph/0310014. 


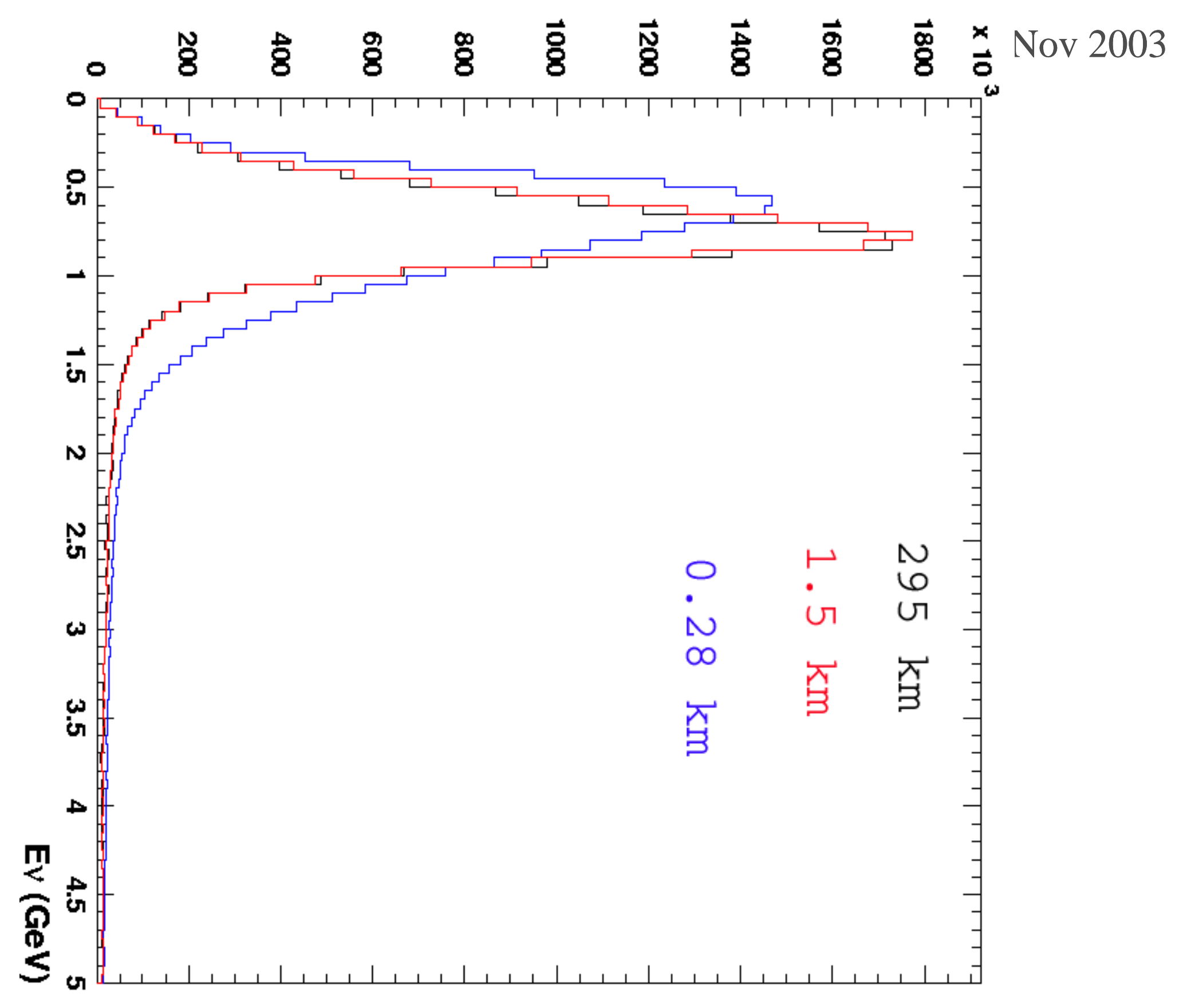

